\title{
Reflexões sobre o conhecimento na formação de professores em comunidade de prática
}

\author{
Francisco Imbernón' (D) @ \\ Alexandre Shigunov Neto ${ }^{2}$ (D) @ \\ André Coelho da Silva ${ }^{3}$ (D) @ \\ ${ }^{1}$ Universidade de Barcelona (España); ${ }^{2}$ Instituto Federal de Educação, Ciência e Tecnologia de São Paulo (IFSP) - \\ Itapetininga (Brasil).
}

Resumo. Assumindo que a criação, a gestão e o compartilhamento do conhecimento são instrumentos primordiais da cidadania, da educação e da nova economia, objetivamos pensar questões associadas a seus processos de criação e compartilhamento no contexto da formação continuada de professores em comunidades de prática. Para isso, apoiamo-nos em noções desenvolvidas no campo da gestão do conhecimento, especialmente na chamada espiral do conhecimento. Com base nela e em estudos relativos aos conceitos e tipos de comunidade, procuramos por meio de um esforço teórico-exploratório descrever/explicar as dinâmicas de (re)construção de conhecimentos decorrentes das interações ocorridas entre professores nas comunidades de prática. Em síntese, apontamos que uma comunidade de prática, ao proporcionar o compartilhamento e a externalização dos conhecimentos tácitos dos professores, pode atuar em prol da transformação desses em conhecimentos explícitos.

Palavras-chave: conhecimento; comunidades de prática; formação continuada de professores.

Reflexiones sobre el conocimiento en la formación de docentes en comunidades de práctica Resumen. Teniendo en cuenta que la creación, la gestión y la coparticipación en el conocimiento son herramientas clave de la ciudadanía, la educación y la nueva economía, pretendemos plantear cuestiones asociadas a sus procesos de creación y coparticipación en el contexto de la formación continua del profesorado en comunidades de práctica. Para ello, nos hemos fundamentado en nociones desarrolladas en el campo de la gestión del conocimiento, en particular, en la espiral de creación del conocimiento. En base a ella y en estudios relacionados con conceptos y tipos de comunidades, buscamos, por medio de un esfuerzo teórico y exploratorio, describir/explicar las dinámicas de (re)construcción de conocimientos resultantes de las interacciones que ocurren entre profesores en las comunidades de práctica. En resumen, señalamos que cuando una comunidad de práctica favorece la coparticipación y la externalización de los conocimientos tácitos de los profesores, puede actuar favorablemente para convertirlos en conocimientos explícitos.

Palabras clave: conocimiento; comunidades de práctica; formación continua de profesores.

\section{Reflections on knowledge in teacher training through communities of practice}

Abstract: Assuming that the creation, management and sharing of knowledge are primordial instruments of citizenship, education and the new economy, we aim to think about issues associated with their creation and sharing processes in the context of continuing teacher training in communities of practice. To do this, we based on notions developed in the field of knowledge management, especially in the so-called knowledge spiral. Based on this and on studies related to concepts and types of community, through a theoretical-exploratory effort, we seek to describe/ explain the dynamics of (re)construction of knowledge arising from interactions between teachers in communities of practice. In summary, we point out that a community of practice, by providing the sharing and externalization of teachers' tacit knowledge, can act in favor of transforming these into explicit knowledge.

Keywords: knowledge; communities of practice; continuing teacher training. 


\section{Considerações iniciais}

Muitos autores caracterizam o momento atual de nossa sociedade como um momento de transição: de uma sociedade industrial para uma sociedade do conhecimento. Este, enquanto novo fator de produção, passa a se configurar como motor da economia e principal fonte de riqueza. Além disso, enquanto recurso, o conhecimento apresenta algumas peculiaridades: é infinito/ilimitado - e não escasso como os recursos naturais e o capital; e cresce à medida que é estimulado e utilizado - ao contrário dos ativos materiais que se depreciam quando são utilizados (Davenport e Prusak, 1998; Stewart, 1998; Drucker, 2002; Castells, 1999; Hargreaves, 2003).

De fato, a informação e o conhecimento parecem estar adquirindo cada vez mais importância no escopo da cadeia produtiva em comparação com os tradicionais fatores de produção, os aspectos associados à mão de obra, os recursos naturais e o capital. Nesse sentido, a relevância do conhecimento na economia e na sociedade como um todo acaba por definir as bases da chamada sociedade do conhecimento.

A temática do conhecimento, enquanto objeto de estudo, é muito antiga e caminha de mãos dadas com a própria história da humanidade. Os primeiros relatos que se têm registro apresentam os filósofos gregos como os pioneiros a se dedicarem ao estudo do conhecimento humano. Com o passar do tempo, em função da importância que adquiriu nas sociedades moderna e contemporânea, a temática do conhecimento se tornou objeto de estudo em diversas áreas (Drucker, 2002; Castells, 1999; Hargreaves, 2003).

A chamada sociedade do conhecimento, de forma geral, e a gestão do conhecimento, de forma específica, têm sido objetos de pesquisa de estudiosos brasileiros e internacionais das mais diversas áreas desde o final da década de 1990. Em termos internacionais, destacam-se, entre outras, as pesquisas de Stewart (1998), Sveiby (1998), Davenport e Pruzak (1998) e Nonaka e Takeuchi (1997). Já no âmbito brasileiro, pode-se destacar, entre outras, as pesquisas de Angeloni (2002), Tarapanoff (2001), Ponchirolli (2003), Silva (2004) e Ponchirolli e Fialho (2005).

Entre esses autores, há certo consenso sobre a consideração de que a criação, a gestão, a produção, a difusão e o compartilhamento do conhecimento são instrumentos primordiais da cidadania, da educação e da nova economia. Além disso, esclarece-se que o indivíduo cria seu conhecimento ao reconstruir conhecimentos já existentes, ou seja: não basta ser capaz de reproduzi-los ou copiá-los, é necessário construir sua própria versão sobre eles. 
Os estudos no ambiente organizacional que originaram o campo conhecido como gestão do conhecimento concebem que este é criado por meio de um processo em que interagem dois tipos de conhecimentos: 0 conhecimento tácito e o conhecimento explícito. 0 conhecimento explícito é aquele registrado em algum meio externo ao indivíduo, como arquivos magnéticos, sistemas de informação e/ou impressão em papel. Já o conhecimento tácito é aquele que está disponível somente na mente do indivíduo, sendo exteriorizado por necessidades contextuais, espontâneas ou dirigidas. O processo de criação do conhecimento passa, portanto, pela conversão dos conhecimentos tácitos em conhecimentos explícitos, o que, no âmbito das organizações, funciona como forma de converter os bens intelectuais em riqueza e vantagem competitiva - para isso, em alguns casos, recorre-se a técnicas e recursos de Tecnologia da Informação e Comunicação (TIC) (Polanyi, 1966; Wiig, 1995; Nonaka; Takeuchi, 1997; Davenport e Pruzak, 1998).

Um dos objetivos da gestão do conhecimento é propiciar condições para a criação de ambientes em que as pessoas busquem e se sintam incentivadas a compartilhar seus conhecimentos, transformando seus conhecimentos tácitos em conhecimentos explícitos (Nonaka; Takeuchi, 1997; Angeloni, 2002; Terra, 2001; Silva, 2004; Ponchirolli \& Fialho, 2005).

Nesse ponto, há que se observar que o compartilhamento de conhecimentos é fundamental para a sobrevivência e o desenvolvimento das organizações - entendidas aqui como qualquer conjunto de pessoas unidas em busca de objetivos em comum. Dessa forma, enquanto organizações educacionais, para as escolas, as universidades e os grupos de estudo e pesquisa, por exemplo, também é fundamental o compartilhamento de conhecimentos.

Pesquisadores que trabalham no campo da gestão do conhecimento consideram que a interação entre as duas formas de conhecimento (o tácito e o explícito) é um processo social entre indivíduos que pode ser sistematizado por meio de uma espiral, a chamada espiral do conhecimento (figura 1, a seguir). Nonaka e Takeuchi (1997), os idelizadores da espiral do conhecimento, entendem que a transformação do conhecimento tácito em conhecimento explícito ocorre em quatro etapas: a socialização, a externalização, a combinação e a internalização.

A socialização é o processo de compartilhamento dos conhecimentos tácitos dos indivíduos, ocorrendo durante a interação entre pares. A externalização é a conversão do conhecimento tácito em conhecimento explícito, momento em que o conhecimento individual é transformado em conhecimento articulado e transmissível para seus pares. A combinação se refere à possibilidade de articulação entre diferentes conhecimentos explícitos, produzindo, assim, novos conhecimentos explícitos. Esse processo pode 
ocorrer quando indivíduos combinam ou trocam conhecimentos por diversas formas, tais como: emails, reuniões, documentos, conversas e interações no âmbito de comunidades de prática. A última etapa da espiral é a conversão do conhecimento explítico em conhecimento tácito. Ela ocorre por meio da interpretação dos conhecimentos explícitos presentes nos documentos, livros etc., levando os indivíduos a os internalizarem e, consequentemente, a reorganizarem seus conhecimentos tácitos (Wiig, 1994 e 1995; Nonaka; Takeuchi, 1997; Davenport e Pruzak, 1998; Terra, 2001).

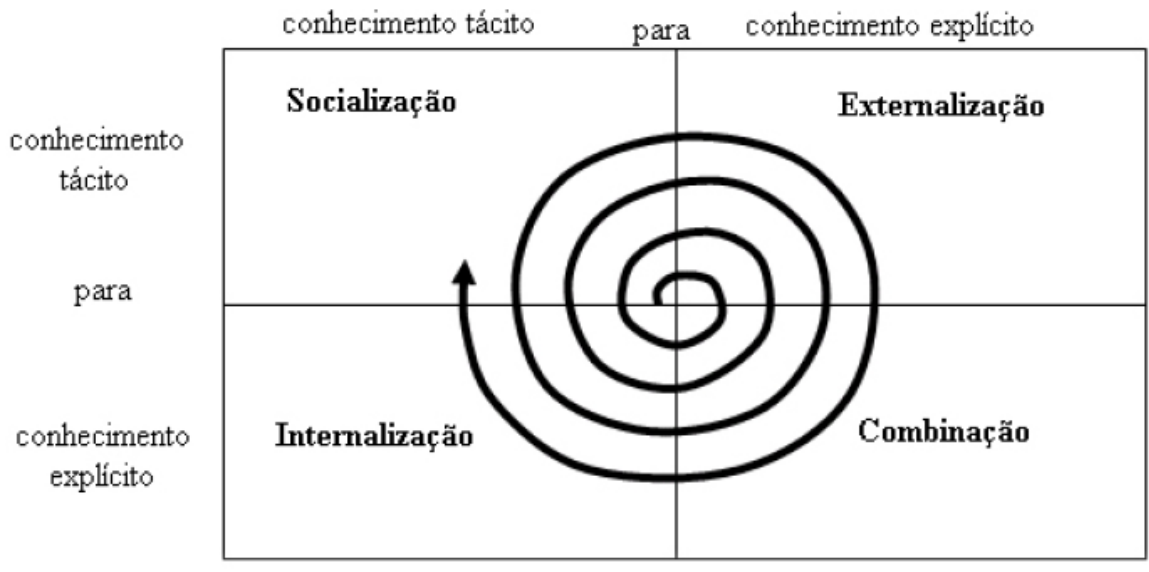

Figura 1: Espiral do conhecimento (Nonaka e Takeuchi, 1997, p. 80).

Tomando como apoio as noções associadas à espiral e assumindo que os processos de ensino e aprendizagem implicam na construção e reconstrução do conhecimento, neste trabalho tecemos considerações a respeito da formação continuada de professores. Em específico, objetivamos realizar reflexões sobre o processo de criação e compartilhamento de conhecimento no contexto das chamadas comunidades de prática.

Para atingirmos o objetivo proposto, nas duas próximas seções procuramos caracterizar alguns tipos de comunidade e de que forma elas vêm sendo pensadas no contexto educacional. Já nas duas últimas seções, tecemos algumas considerações sobre a utilização das comunidades de prática como instrumento auxiliar na formação continuada de professores.

A estratégia metodológica empregada no desenvolvimento deste trabalho tem como base o estudo da literatura no que se refere: ao conceito, à criação e ao compartilhamento do conhecimento; ao conceito de comunidade e às características associadas às comunidades formativa, de aprendizagem e de prática; e à utilização de comunidades de prática junto à formação continuada de professores. A pretensão não foi realizar um extenso e rigoroso 
estado da arte sobre tais assuntos. Ao invés disso, tomamos como base de reflexão elementos trazidos pela literatura - especialmente a ideia da espiral do conhecimento e os processos a ela associados -, a fim de oferecermos um novo olhar sobre as dinâmicas de (re)construção de conhecimentos decorrentes das interações ocorridas entre professores nas comunidades de prática. Trata-se, assim, de um esforço teórico-exploratório que visa descrever/ explicar tais dinâmicas.

\section{Comunidades formativa e de aprendizagem - um olhar focado no campo da Educação}

Uma comunidade formativa (às vezes designada de educação comunitária) difere em alguns aspectos de uma comunidade de aprendizado. A comunidade formativa ocorre em contextos que permitem aos professores desenvolver sua própria cultura dentro do grupo e não apenas a reprodução padronizada da cultura social ou acadêmica dominante. É um contexto relevante para a (re)elaboração, pelos próprios membros, de suas concepções e práticas de ensino e aprendizagem, com base nas tarefas realizadas, nas experiências e nas interações experimentadas. As instituições educacionais podem funcionar como comunidade formativa na medida em que geram múltiplas situações de comunicação e uso das linguagens oral e escrita no contexto dos relacionamentos interpessoais.

As pessoas que compõem uma comunidade formativa desempenham papéis ativos na construção e reelaboração de valores, regras de relacionamento e normas sociais e educativas. O respeito pela diversidade é estimulado. Nesses contextos formativos, destacam-se processos associados à elaboração e à internalização de conceitos fundamentais para a organização da aprendizagem: o valor de uso do tempo e sua organização, o uso dos espaços e as noções de trabalho, ensino e aprendizagem (Torres, 2006).

Entre as finalidades de uma comunidade formativa está a construção de um projeto educacional comunitário que enfatize a cooperação e a solidariedade. Parte-se da ideia de que os recursos que a instituição possui devem ser valorizados e articulados para criar um projeto educacional comunitário associado às necessidades e às possibilidades específicas do coletivo. De fato, ao considerarmos que nessas comunidades formativas o protagonismo é assumido pelos professores, podemos designá-las de comunidades formativas de professores. 
Outro tipo de comunidade abordado pela literatura da área são as chamadas comunidades de aprendizagem (ou comunidades de aprendizado). Essas comunidades, no contexto da educação, têm como objetivo principal transformar a escola em um agente efetivo de transformação social. Paralelamente a isso, espera-se fomentar a aprendizagem dos alunos e a elevação de suas autoestimas. Embora a experiência de transformar as escolas em comunidades de aprendizagem leve tempo, defende-se que o incentivo ao diálogo, à participação, à cooperação e à solidariedade entre todos os envolvidos pode trazer impactos positivos à comunidade como um todo.

Alguns princípios estruturam as comunidades de aprendizagem: I) Todos os membros compartilham objetivos e, nesse sentido, o processo de ensino e aprendizagem não está unicamente nas mãos dos professores. A participação das famílias, das associações de bairro, de voluntários, de instituições sociais etc., é entendida como fundamental. II) 0 ambiente de aprendizagem é organizado de maneira alternativa à escola tradicional. Logo, as atividades não são desenvolvidas em salas de aula fechadas, onde cada professor é responsável pela aprendizagem de um grupo de alunos. Valoriza-se a participação de mais adultos e o trabalho ativo dos alunos. III) A escola é entendida como o centro de aprendizagem para toda a comunidade. IV) Os processos de ensino são planejados tendo em vista objetivos coletivos. V) Expectativas elevadas são criadas (ao invés de metas mínimas) e recorre-se a todos os meios disponíveis para alcançá-las. VI) A realização de atividades cooperativas em grupo é incentivada, dando espaço ao uso de diferentes habilidades pelos estudantes. VII) A avaliação é realizada de forma contínua e sistemática por toda a comunidade. VIII) 0 nível de compromisso e de participação dos alunos, da família e de toda a comunidade é alto. IX) A liderança escolar é compartilhada e a coordenação de todo o trabalho é realizada por meio de comissões em que participam professores, mães, pais, estudantes, administração, voluntariado etc. X) A relação de cooperação entre os alunos e a atuação de grupos mais experientes como tutores de grupos menos experientes é valorizada. Nesse sentido, incentiva-se a formação de turmas flexíveis e heterogêneas.

Ao assumirmos que uma comunidade diz respeito mais a um conjunto compartilhado de práticas do que às estruturas envolvidas (Bourdieu, 1991), os entendimentos sobre as noções de escola e sala de aula e sobre as possibilidades e funções educacionais desses espaços acabam se expandindo. Uma comunidade não se resume a um lugar, a algo físico, pois se refere à criação de um campo discursivo, o que implica no desenvolvimento de práticas comunicativas. Em outras palavras: uma comunidade é alimentada e organizada pela comunicação e pelo diálogo e não pela autoridade, pelo status ou pelo rito (Habermans, 1998). Assim, os membros de uma comunidade - como os estudantes, num contexto escolar - possuem papel 
ativo e autonomia na estruturação de referências e regulamentações para as práticas a ela associadas, tanto no que se refere a questões como tempos e espaços, quanto no que diz respeito a estilos comunicativos.

Do ponto de vista formativo, trabalhar em conjunto com a comunidade é uma proposta orientada para a construção de cenários educativos mais inovadores dentro da instituição de ensino, por meio da participação de vários agentes sociais que compõem a comunidade: os alunos, os profissionais da escola, as famílias etc. Cada um desses agentes pode contribuir com elementos valiosos para a educação, o que contribui para o desenvolvimento pessoal/profissional de todos.

Já do ponto de vista acadêmico, o trabalho em comunidades pode proporcionar aos participantes o desenvolvimento de suas habilidades socioafetivas, cognitivas e psicolinguísticas, uma vez que se ampliam as oportunidades de interação social, comunicação oral e escrita e prática na resolução de problemas de vários tipos.

\section{Comunidades de prática: origem e conceitos}

Nos anos finais do século XX, parece ter sido consolidada no campo educacional a ideia de que, em certos contextos sociais, não faz sentido considerar que a educação pode/deve agir como salvadora de pessoas em situação de exclusão social. Os atuais contextos sociais, familiares e econômicos evidenciam que, sem a ajuda da comunidade que envolve a instituição educativa, dificilmente os estudantes adquirem aptidões, habilidades e competências - como senso democrático, social, paritário, intercultural e ambiental -, que, a princípio, possibilitam uma vida e um mundo melhor. A velha ideia de "viver feliz" dificilmente pode ser oferecida de maneira isolada pela educação. E é, nesse contexto, que o conceito de comunidade retorna de uma maneira diferente.

A partir da década de 1990, a temática das chamadas comunidades de prática (CP) tornou-se objeto de estudo de pesquisadores internacionais, com destaque para os estudos de Lave e Wenger (1991), Wenger (1998) e Wenger, McDermontt e Snyder (2002). De fato, pode ser atribuída aos pesquisadores Jean Lave e Etienne Wenger a paternidade desse conceito:

Os estudos iniciais davam destaque à relação entre estudantes e professor como fonte primordial de aprendizado. 0 aprofundamento no estudo das relações sociais revelou a Wenger uma maior complexidade a respeito desse assunto. A partir da visão social, evidencia-se a noção de que o 
aprendizado se encontra situado na troca de conhecimentos com pessoas mais experientes e aprendizes mais avançados. Dessa maneira, o termo comunidade de prática passou a ser utilizado, designando a comunidade que opera dinamicamente em todos os lugares, mesmo não existindo uma relação sistemática entre as pessoas. Cabe ressaltar que embora existam inúmeras iniciativas de implementação e consolidação de comunidades de prática, os estudos teóricos têm sido sustentados a partir da visão de Wenger. (Silva, 2004, p. 72)

Analisando a obra de Lave e Wenger, Silva (2004, p. 72-73) caracteriza a visão dos autores em relação às $\mathrm{CP}$ :

Como um grupo de pessoas que mistura relacionamentos e atividades, num determinado tempo e em relação tangencial e envolvimento com outras comunidades. Nessas comunidades, os novos membros aprendem com os mais velhos ao serem autorizados a participar de certas práticas da comunidade. Os autores afirmam que "veem as comunidades de prática como uma condição intrínseca para a existência do conhecimento". Eles perceberam que a aprendizagem em tais comunidades não é simplesmente uma reprodução de conhecimentos, mas sim uma aprendizagem com a participação legítima de seus membros.

Logo, uma CP não é apenas uma reunião, um conjunto de pessoas que possuem afinidades pessoais e/ou profissionais. Tampouco pode ser confundida como sinônimo de grupo, time ou rede de pessoas. Há diferenças e peculiaridades que distinguem os grupos/equipes das CP. Nestas: I) não existe uma hierarquia formal; II) a legitimação dos participantes é informal; III) as pessoas compartilham os conhecimentos em função de afinidades profissionais e de áreas de estudo específicas; IV) o status do participante é regulado em função de seus conhecimentos (Wenger, 1998; Silva, 2004; Imbernón, 2010).

As CP são comunidades em que os indivíduos têm a oportunidade de aprender, pois criam, gerenciam e compartilham conhecimentos. Para que efetivamente ocorra, Wenger (1998) aponta a necessidade de que haja envolvimento e compromisso mútuo entre os participantes, bem como um repertório linguístico comum a todos - o que possibilitaria o compartilhamento dos significados e dos conhecimentos.

Wenger (1998) e Silva (2004) distinguem três características fundamentais para a existência das CP, características essas que podemos denominar de "tripé das comunidades de prática": I) conhecimento - o interesse em criar e compartilhar conhecimentos sobre determinadas temáticas é o que motiva os indivíduos a participarem e criarem as comunidades de prática; II) comunidade/membros - o conceito de grupo proporciona que os indivíduos constituam um vínculo e um relacionamento em torno de conhe- 
cimentos específicos; e III) prática - os membros de uma CP compartilham interesses comuns em torno de determinados conhecimentos. As experiências profissionais em determinada área de conhecimento costumam funcionar como vínculo para os integrantes de uma CP, os quais, em geral, pertencem a uma mesma organização.

Embora uma CP seja um grupo constituído para desenvolver conhecimento especializado, ela não pode ser considerada uma comunidade científica, visto que seu funcionamento tem como base a comunicação e a reflexão sobre experiências práticas vivenciadas pelos participantes. Sendo assim, numa CP, baseados no contexto e na reflexão sobre suas experiências práticas, os indivíduos definem os objetivos de aprendizagem do grupo.

Por fim, é pertinente mencionar que a rede de conhecimento criada no interior de uma CP leva em conta a cultura, os valores e as crenças de seus membros e da própria organização, ou seja, apesar de existirem características primordiais que devem estar presentes em qualquer $\mathrm{CP}$, podem haver variações em relação a seus modos de funcionamento e atuação - por exemplo: os encontros das CP podem ser feitos presencialmente ou virtualmente.

\section{Comunidades de prática na formação de professores}

Conforme nossos apontamentos anteriores, na educação e, em específico, na formação de professores, o conceito de comunidade vem sendo utilizado e complementado de diferentes maneiras: comunidade de prática; comunidade de conhecimento; comunidade de aprendizagem; comunidade investigativa; entre outros. Pesquisadores como Cochran-Smith e Lytle (2002), Elbo, Puigdellívol, Soler e Valls (2002), Delante (2006), Fiorentini (2010, 2013), Creci e Fiorentini (2013) e Imbernón (2009, 2010) têm se apropriado desse conceito em suas pesquisas visando a proposição de alternativas para o processo de formação de professores.

Creci e Fiorentini (2013) consideram que o surgimento de grupos colaborativos na década de 1990 decorreu da mudança em relação a como o processo de formação continuada de professores é entendido. Este teria passado a ser visto como um processo complexo que vai além da aplicação de teorias e estudos acadêmicos. Nesse sentido, passa-se a assumir que o desenvolvimento profissional, a aprendizagem e a formação continuada dos professores como um todo podem ocorrer também em CP. 
Para Fiorentini (2010), os estudos sobre a formação de professores e, em específico, sobre a formação continuada de professores, evidenciam que a complexidade da prática docente num mundo em constantes mudanças requer que o aprendizado seja contínuo. 0 aprendizado e o desenvolvimento profissional contínuo dos professores prima pela prática pedagógica e, consequentemente, instrumentos como as CP têm sido desenvolvidos para fomentá-los.

No âmbito da formação continuada de professores, podemos afirmar que, em síntese, uma CP funciona como um grupo de professores que trocam, refletem e aprendem uns com os outros tomando como matéria-prima relatos sobre suas práticas. Dessa forma, consideramos que as $\mathrm{CP}$ podem contribuir para a criação e o compartilhamento do conhecimento dos professores, especialmente porque parte dele é construído por meio da prática, configurando-se como conhecimento tácito, não formalizado. Através do processo sistematizado na espiral apresentada na figura 1 , as $\mathrm{CP}$, na medida em que possibilitam 0 compartilhamento do conhecimento, favorecem a transformação do conhecimento tácito dos professores em conhecimento explícito, proporcionando também a internalização e a recriação deste.

\section{Algumas palavras finais}

As pesquisas realizadas apontam a pertinência em se fazer uso de comunidades de prática no âmbito da formação continuada e permanente de professores, contribuindo de forma efetiva para o compartilhamento e a construção de novos conhecimentos oriundos de experiências vivenciadas por meio da prática escolar.

Ao funcionarem como um grupo de professores que trocam, refletem e aprendem uns com os outros a partir de suas práticas, as comunidades de prática podem ser caracterizadas como um instrumento auxiliar importante voltado a suas formações continuadas. Conforme esperamos ter evidenciado, o conhecimento construído por meio da prática configura-se como conhecimento tácito, não formalizado. Assim, a partir do estabelecimento de diálogos e trocas de experiência no escopo das comunidades de prática, tais conhecimentos tácitos podem ser externalizados, debatidos e transformados em conhecimentos explícitos. 


\section{Referências bibliográficas}

Angeloni, M. T. (Org.) (2002). Organizações do conhecimento: infraestrutura, pessoas e tecnologia. São Paulo: Saraiva.

Bourdieu, P. (1991). El sentido práctico. Madrid: Taurus.

Castells, M. (1999). A Era da Informação: economia, sociedade e cultura, vol. 3. São Paulo: Paz e Terra.

Cochran-Smith, M. y Lytle, S. (2002). Dentro/fuera. Enseñantes que investigan. Madrid: Akal.

Crecci, V. M. e Fiorentini, D. (2013). Desenvolvimento Profissional de Professores em Comunidades com Postura Investigativa. Acta Scientiae Canoas, 15(1), 9-23.

Davenport, T. H. e Prusak, L. (1998). Conhecimento Empresarial. Rio de Janeiro, Campus.

Delante, G. (2006). Community. Comunidad, educación ambiental y ciudadanía. Barcelona: Graó.

Drucker, P. (2002). Sociedade pós-industrial. São Paulo: Pioneira Thomson Learning.

Elbo, C., Puigdellívol, I., Soler, M. \& Valls, R. (2002). Comunidades de aprendizado. Transformar a educação. Barcelona: Graó.

Fiorentini, D. (2010). Desenvolvimento profissional e comunidades investigativas. In: Dalben, Â.; Diniz, J.; Leal, L.; Santos, L. (Orgs.). Convergências e tensões no campo da formação e do trabalho docente: educação ambiental - educação em ciências - educação em espaços não escolares - educação matemática p. 570-590. Belo Horizonte: Ática.

Fiorentini, D. (2013). Learning and professional development of mathematics teacher in research communities. Sisyphus - Journal of Education, 1(3), 152-181.

Habermas, J. (1998). Teoria da ação comunicativa. I. Racionalidade de ação e racionalização social. II. Crítica da razão funcionalista. Madrid: Touro.

Hargreaves, A. (2003). O Ensino na Sociedade do Conhecimento: a educação na era da insegurança. Colecção Currículo, Políticas e Práticas. Porto: Porto Editora.

Imbernón, F. (2010). A formación permanente a través de redes de profesorado. Eduga: revista galega do ensino, 60.

Imbernón, F. (2009). Formação permanente do professorado: novas tendências. São Paulo: Cortez.

Lave, J. \& Wenger, E. (1991). Situated learning: legitimate peripheral participation. Cambridge: Cambridge University Press.

Nonaka, I. \& Takeuchi, H. (1997). Criação de Conhecimento na Empresa. Rio de Janeiro: Campus.

Polanyi, M. (1966). The tacit dimension. London: Routdedge \& Kegan Paul.

Ponchirolli, O. e Fialho, F.A.P. (2005). Gestão estratégica do conhecimento como parte da estratégia empresarial. Revista FAE, 8(1), 127-138.

Ponchirolli, O. (2003). A importância do capital humano na gestão estratégica do conhecimento sob a perspectiva da teoria do agir comunicativo. Tese de Doutorado. Programa de Pós-Graduação em Engenharia da Produção. UFSC. 
Silva, H. de F. N. (2004). Criação e compartilhamento de conhecimento em comunidades de prática: uma proposta metodológica. Tese de Doutorado. Programa de Pós-Graduação em Engenharia da Produção, UFSC, Florianópolis.

Stewart, T. A. (1998). Capital Intelectual: A Nova Vantagem Competitiva das Empresas. Rio de Janeiro: Campus.

Sveiby, K. E. (1998). A Nova Riqueza das Organizações. Rio de Janeiro: Campus.

Tarapanoff, K. (Org.) (2001). Inteligência organizacional e competitiva. Brasília: Editora da UNB.

Terra, J.C.C. (2001). Gestão do Conhecimento. São Paulo: Negócios.

Torres, J. (2006). La desmotivación del profesorado. Madrid: Morata.

Wenger, E. (1998). Communities of practice: learning, meaning, and identity. Cambridge: Cambridge University Press.

Wenger, E., Mcdermontt, R. \& Snyder, W. M. (2002). Cultivating communities of practice. Boston: Harvard Business School Press.

Wiig, K. M. (1994). Knowledge Management: The Central Management Focus for IntelligentActing Organizations. Arlington, TX: Schema Press.

Wiig, K. M. (1995). Knowledge Management Methods: Practical Approaches to Managing Knowledge. Arlington, TX: Schema Press. 